\title{
Quantization of gravity in spherical harmonic basis
}

\author{
Renata Kallosh $\odot^{*}$ \\ Stanford Institute for Theoretical Physics and Department of Physics, Stanford University, \\ Stanford, California 94305, USA
}

(Received 9 September 2021; accepted 24 September 2021; published 19 October 2021)

\begin{abstract}
We perform canonical quantization of gravity in the background of a Schwarzschild black hole in the generalized Regge-Wheeler gauge proposed in Kallosh and Rahman [Quantization of gravity in the black hole background, Phys. Rev. D 104, 086008 (2021)]. We find that the Hamiltonian at the quadratic level is unitary and ghost-free. Two canonical degrees of freedom are associated with Zerilli-Moncrief and Cunningham-Price-Moncrief functions of the metric perturbations. The $\ell<2$ part of the Hamiltonian vanishes. This quantization with the unitary Hamiltonian for gravity is valid also in Minkowski space in spherical coordinates.
\end{abstract}

DOI: 10.1103/PhysRevD.104.086023

\section{INTRODUCTION}

This article is a development based on a recent paper [1] where we have performed a covariant (Lagrangian) quantization of gravity in a black hole background in the ReggeWheeler setup [2-4]. The gauge-fixing condition in [1] includes the Regge-Wheeler gauge for $\ell \geq 2$ modes and a certain background covariant gauge for $\ell<2$ modes, where the Regge-Wheeler gauge is not valid. We will refer to the gauge in [1] covering all $\ell$ modes as a "generalized Regge-Wheeler gauge."

The Feynman path integral for gravity, viewed as quantum field theory (QFT), is defined by De WittFaddeev-Popov [5,6] and takes a form, in absence of sources,

$$
\int D h J_{\chi}(g, h) \delta\left(\chi_{\alpha}(g, h)\right) e^{\mathrm{i} S(g+h)}
$$

Here we integrate over the perturbations $h$ in the background metric $g$. The gauge-fixing conditions are $\chi_{\alpha}(g, h)=0$. The Jacobian designed to make this path integral independent on the choice of the gauge-fixing conditions can be presented with the help of the FaddeevPopov (FP) ghosts [6]

$$
J_{\chi}=\int D \bar{C}^{\alpha} D C_{\beta} e^{\mathrm{i} \int \mathrm{d}^{4} x \bar{C}^{\alpha}(x) Q_{\alpha}^{\beta}(g, h) C_{\beta}(x)} .
$$

\footnotetext{
*kallosh@stanford.edu
}

Published by the American Physical Society under the terms of the Creative Commons Attribution 4.0 International license. Further distribution of this work must maintain attribution to the author(s) and the published article's title, journal citation, and DOI. Funded by SCOAP ${ }^{3}$.
The differential operator in the ghost action is defined by the gauge variation of the gauge-fixing functions $\delta \chi_{\alpha}=Q_{\alpha}^{\beta} \xi_{\beta}$. For the choice of the gauge-fixing functions made in [1], which in addition to the Regge-Wheeler gauge for $l \geq 2$ modes includes gauges for $l<2$ modes, a generalized Regge-Wheeler gauge, we have found that the ghost actions do not have time derivatives in Schwarzschild coordinates. We therefore predicted that in the generalized Regge-Wheeler gauge [1] the canonical Hamiltonian according to the rules for gauge theories [7-9] is expected to be unitary.

In this paper we will present the quadratic in the gravitational perturbations $h$ part of the gravity Hamiltonian in the spherical harmonic basis. Before doing this we will perform the standard counting of physical degrees of freedom in this case. The structure of the Hamiltonian will confirm this counting.

The standard counting of physical degrees of freedom in gauge theories in the QFT context of the Feynman path integral is the same in either Lagrangian or Hamiltonian quantization, and it is also gauge independent, if performed correctly. The general counting formula is formulated for the number of gauge field components equal to $n+k$ in the case of $k$ gauge symmetries. The total number of physical degrees of freedom is

$$
n-k \text {. }
$$

This final counting formula in QFT is valid for any choice of gauge fixing, but the procedure is different for unitary and pseudo-unitary gauges. For example, in four dimensions the metric has $n+k=10$ components and there are $k=4$ gauge symmetries; the counting is $n-k=(10-4)-4=2$. 
In QFT in the class of unitary gauges Hamiltonians have manifestly ghost-free underlying Hilbert spaces. There are $\left(p^{*}, q^{*}\right)$ variables in Faddeev's theorem [7] as described in [1]. This means that all $n-k$ physical states have a positive definite metric. The $S$ matrix is unitary.

$$
\text { \#degrees of freedom } \text { unitary } \mathrm{H}=n-k \text {. }
$$

Meanwhile, in other gauges, for example, 4D Lorentz covariant gauges in gravity, the Hamiltonians are "pseudounitary" with underlying state spaces with negative-norm ghost degrees of freedom [10,11]. In such a case the counting goes as follows: there are $n+k$ states with positive norm and $2 k$ states with negative norm presented by FP anticommuting ghosts, so the total counting, with the account of negative norm states, is the same as in unitary gauges

\#degrees of freedom $_{\text {pseudo-unitary } \mathrm{H}}=n+k-2 k \Rightarrow n-k$.

The $S$ matrix is pseudo-unitary in a space of states with the indefinite metric.

We will see that the quadratic in the $h$ part of the gravity Hamiltonian in spherical harmonic basis does support this counting. In the class of gauges used in [1] the canonical Hamiltonian is unitary, as predicted there.

The complete form of the Hamiltonian to all orders of $h$ is beyond the scope of this paper. However, in [1] we have argued that the nonlinear couplings of ghosts to all orders in $h$ are free of time derivatives on ghosts. Therefore one would expect that the nonlinear in $h$ terms in $H$ will be consistent with the unitarity of the Hamiltonian which will be deduced in this article at the level quadratic in $h$.

The corresponding part of the action $S(g+h)$, quadratic in $h$, is of the form

$$
S=\frac{1}{2} \int h_{\mu \nu} S^{\mu \nu \lambda \delta}(g) h_{\lambda \delta} .
$$

Here $S^{\mu \nu \lambda \delta}(g)$ is a differential operator depending on the background metric $g$. The left-hand side of equations of motion $\frac{\delta S}{\delta h_{\mu \nu}}=0$ linear in $h$ takes the form

$$
Q^{\mu \nu} \equiv \frac{\delta S}{\delta h_{\mu \nu}}=S^{\mu \nu \lambda \delta}(g) h_{\lambda \delta}
$$

One can restore the action in Eq. (1.6) from the information available in Eq. (1.7).

In the spherical harmonic basis the $4 \mathrm{D}$ spacetime is split into $\mathcal{M}=\mathcal{M}_{2} \times \mathbb{S}^{2}$ with coordinates $\left(x^{a}, \theta^{A}\right), a=1,2$. The 4D perturbations $h_{\mu \nu}$ are represented by 2D fields for each $(\ell, m)$ [2-4]. The corresponding equations are known, and we will use them as derived in [4] in Schwarzschild coordinates. Once the quadratic Lagrangian is known, it is possible to derive the relevant quadratic in $h$ Hamiltonian. For $\ell \geq 2$ modes the corresponding quadratic Hamiltonian was constructed by Moncrief in [12] where also the relevant Regge-Wheeler [2] and Zerilli [3] equations were rederived in the form of Hamiltonian equations of motion. The Hamiltonian was derived in [12] in the absence of source terms. For $\ell<2$ the Hamiltonian was not studied, to the best of our knowledge. In [12] it was explained that the attention was restricted to modes with $l \geq 2$ since the modes with $l<2$ are nonradiative and require a special treatment.

Here we will use the known field equations (1.7) in the form given in [4] in Schwarzschild coordinates, which allow one to derive the Lagrangian in (1.6). From the quadratic Lagrangian we derive a canonical quadratic part of the Hamiltonian, with an account of the algebraic constraints in our gauges. We will conclude that there are no physical degrees of freedom suitable for quantization at $\ell<2$. Our definition of quantized degrees of freedom involves the QFT quantization conditions in 2D space of the form

$$
\left[q(r, t), p\left(r^{\prime}, t\right)\right]=i \delta\left(r-r^{\prime}\right) .
$$

The classical field equations for low multipoles in the presence of sources are known to have nontrivial solutions. For example, for the monopoles $\ell=m=0$ there are solutions such as $h_{t t}^{00} \sim \frac{\delta M}{r}$, and they are known to affect the black hole mass. However, there are no solutions of the constraint equations compatible with the quantization condition (1.8) for $\ell<2$.

All our results are valid for any mass $M$ of the Schwarzschild black hole, and the limit to $M=0$ is continuous. This means that they apply not only to the quantization in the black hole background but also to the unitary quantization of the gravitational field in the Minkowski space background in spherical coordinates.

\section{COUNTING GRAVITY PHYSICAL DEGREES OF FREEDOM IN THE SPHERICAL HARMONIC BASIS}

The ansatz of Regge-Wheeler for the metric perturbations $h_{\mu \nu}$ with spherical harmonics of definite parity is given in [2-4]. In our recent paper [1] it was adapted for the purpose of quantization following the formalism and notations in [4]. In particular, we have presented the gauge symmetry transformations to all orders in $h$. The background metric in Schwarzschild coordinates is 


$$
\begin{aligned}
g_{\mu \nu} d x^{\mu} d x^{\nu} & =-f(r) \mathrm{d} t^{2}+\frac{\mathrm{d} r^{2}}{f(r)}+r^{2}(x) \mathrm{d} \Omega_{2}^{2}, \\
f(r) & =1-\frac{2 G M}{r} .
\end{aligned}
$$

The 2D fields representing all components of $h_{\mu \nu}$ in 4D include the following:

$h_{a b}^{\ell m(+)}, \quad j_{a}^{\ell m(+)}, \quad K^{\ell m(+)}, \quad G^{\ell m(+)} \quad \ell>1, \quad$ even,

$h_{a}^{\ell m(-)}, \quad h_{2}^{\ell m(-)} \quad \ell>1, \quad$ odd,

$h_{a b}^{1 m(+)}, \quad j_{a}^{1 m(+)}, \quad K^{1 m(+)} \quad \ell=1, \quad$ even,

$h_{a}^{1 m(-)} \quad \ell=1, \quad$ odd,

$h_{a b}^{00(+)}, \quad K^{00(+)} \quad \ell=0, \quad$ even.

The gauge symmetries are also expanded in spherical harmonics. In the form given in our recent paper [1] these are

$$
\begin{aligned}
& \xi^{\ell>1} \text { even } \Rightarrow\left\{\xi_{a}^{\ell m(+)}, \xi^{\ell m(+)}\right\}, \\
& \xi^{\ell>1} \text { odd } \Rightarrow\left\{\xi^{\ell m(-)}\right\}, \\
& \xi^{\ell=1} \text { even } \Rightarrow\left\{\xi_{a}^{1 m(+)}, \xi^{1 m(+)}\right\}, \\
& \xi^{\ell=1} \text { odd } \Rightarrow\left\{\xi^{1 m(-)}\right\}, \\
& \xi^{\ell=0} \text { even } \Rightarrow\left\{\xi_{a}^{00(+)}\right\} .
\end{aligned}
$$

The gauge symmetry parameters $\xi_{a}^{\ell m(+)}, \xi^{\ell m(+)}, \xi^{\ell m(-)}$ can be regarded as scalar and vector fields on $\mathcal{M}_{2}$.

The counting of physical degrees of freedom in these five sectors is as follows:
(1) $\ell>1$ even: $n+k=7, k=3 \Rightarrow n+k-2 k=$ $7-2 \cdot 3=1$,
(2) $\ell>1$ odd: $n+k=3, k=1 \Rightarrow n+k-2 k=$ $3-2 \cdot 1=1$,
(3) $\ell=1$ even: $n+k=6, k=3 \Rightarrow n+k-2 k=$ $6-2 \cdot 3=0$
(4) $\ell=1$ odd: $n+k=2, k=1 \Rightarrow n+k-2 k=$ $2-2 \cdot 1=0$,
(5) $\ell=0$ even: $n+k=4, k=2 \Rightarrow n+k-2 k=$ $4-2 \cdot 2=0$.

Thus we find that in the $l \geq 2$ sector there is one even and one odd physical degree of freedom for each $(\ell, m)$. There are no degrees of freedom for any of $\ell<2$.

\section{QUADRATIC LAGRANGIAN/HAMILTONIAN FOR $\ell \geq 2$ MODES}

\section{A. $\ell \geq 2$ even}

There are seven fields here, $h_{a b}^{\ell m(+)}, j_{a}^{\ell m(+)}$, $K^{\ell m(+)}, G^{\ell m(+)}$. There are seven equations of motion for these fields. Now we can add the three Regge-Wheeler gauge-fixing conditions,

$$
G=j_{a}=0
$$

The remaining four fields are $h_{a b}^{\ell m(+)}, K^{\ell m(+)}$. We expect to identify three constraints that will leave us with just one canonical degree of freedom. These equations are according to [4]

$$
\begin{aligned}
Q^{t t}= & -\frac{\partial^{2}}{\partial r^{2}} K-\frac{3 r-5 M}{r^{2} f} \frac{\partial}{\partial r} K+\frac{f}{r} \frac{\partial}{\partial r} h_{r r}+\frac{(\lambda+2) r+4 M}{2 r^{3}} h_{r r}+\frac{\mu}{2 r^{2} f} K, \\
Q^{t r}= & \frac{\partial^{2}}{\partial t \partial r} K+\frac{r-3 M}{r^{2} f} \frac{\partial}{\partial t} K-\frac{f}{r} \frac{\partial}{\partial t} h_{r r}-\frac{\lambda}{2 r^{2}} h_{t r}, \\
Q^{r r}= & -\frac{\partial^{2}}{\partial t^{2}} K+\frac{(r-M) f}{r^{2}} \frac{\partial}{\partial r} K+\frac{2 f}{r} \frac{\partial}{\partial t} h_{t r}-\frac{f}{r} \frac{\partial}{\partial r} h_{t t}+\frac{\lambda r+4 M}{2 r^{3}} h_{t t}-\frac{f^{2}}{r^{2}} h_{r r}-\frac{\mu f}{2 r^{2}} K, \\
Q^{b}= & -\frac{\partial^{2}}{\partial t^{2}} h_{r r}+2 \frac{\partial^{2}}{\partial t \partial r} h_{t r}-\frac{\partial^{2}}{\partial r^{2}} \tilde{h}_{t t}-\frac{1}{f} \frac{\partial^{2}}{\partial t^{2}} K+f \frac{\partial^{2}}{\partial r^{2}} \tilde{K}+\frac{2(r-M)}{r^{2} f} \frac{\partial}{\partial t} h_{t r}-\frac{r-3 M}{r^{2} f} \frac{\partial}{\partial r} h_{t t} \\
& -\frac{(r-M) f}{r^{2}} \frac{\partial}{\partial r} h_{r r}+\frac{2(r-M)}{r^{2}} \frac{\partial}{\partial r} K+\frac{\lambda r^{2}-2(2+\lambda) M r+4 M^{2}}{2 r^{4} f^{2}} h_{t t}-\frac{\lambda r^{2}-2 \mu M r-4 M^{2}}{2 r^{4}} h_{r r} .
\end{aligned}
$$

Here

$$
\lambda=\ell(\ell+1), \quad \mu=(\ell-1)(\ell+2) .
$$

The quadratic in the $h$ Lagrangian can be restored from these equations as explained in Eqs. (1.6) and (1.7). One can proceed by defining for each of the four fields their canonical momenta. For example, there is no time 
derivative on $h_{t t}$ in the action; therefore $p_{t t}=0$. The other three coordinates in the form of $\mathcal{L}(q, \dot{q})$ do have time derivatives; however, two more combinations of $q$ 's and $p$ 's are constrained. Only one independent canonical degree of freedom out of four is left.

The Hamiltonian of the related system starting with the Arnowitt, Deser, Misner construction was derived in [12]. We skip the details of the derivation here starting with the field equations (3.2) since the answer for the corresponding Lagrangian can also be reconstructed from the ZerilliMoncrief function [3,12] which in the Regge-Wheeler gauge is

$$
\Psi_{\mathrm{even}}=\frac{2 r}{\ell(\ell+1)}\left[K+\frac{2 f}{\Lambda}\left(f h_{r r}-r K_{, r}\right)\right], \quad l \geq 2,
$$

where $\Lambda=(\ell-1)(\ell+2)+6 M / r$. The equation of motion in the form of the Zerilli-Moncrief function $\Psi_{\mathrm{even}}^{l m}$ as given in [4] is

$$
\left(\square-V_{\text {even }}\right) \Psi_{\text {even }}=S_{\text {even }},
$$

where $\square=g^{a b} \mathcal{D}_{a} \mathcal{D}_{b}$ is the Laplacian operator on $\mathcal{M}_{2}$, $V_{\text {even }}$ depends on $r$ as well as on $M$ and on $l$, and $S_{\text {even }}$ is the contribution from sources. We refer to details given in [4], where also the relation between the Zerilli-Moncrief function and the original Regge-Wheeler function is explained. Equation (3.4) can be derived from the Lagrangian of the form (1.6),

$\mathcal{L}=\sum_{\ell \geq 2, m}\left[\frac{1}{2} \Psi_{\text {even }}\left(\square-V_{\text {even }}\right) \Psi_{\text {even }}-\Psi_{\text {even }} S_{\text {even }}\right]$.

This can be rewritten in the form producing a quadratic part of the Hamiltonian. With $\Psi_{\text {even }} \equiv Q_{\text {even }}$ and its canonically conjugate $P_{\text {even }}$, and in the absence of sources,

$$
\begin{aligned}
H_{\ell \geq 2, \mathrm{even}}= & \frac{1}{2} \sum_{\ell \geq 2, m} \int\left[d r f\left(P^{\ell, m}\right)_{\mathrm{even}}^{2}+f\left(Q_{, r}^{\ell, m}\right)_{\mathrm{even}}^{2}\right. \\
& \left.+V_{\mathrm{even}}\left(Q^{\ell, m}\right)_{\mathrm{even}}^{2}\right],
\end{aligned}
$$

where

$V_{\mathrm{even}}=\frac{1}{\Lambda^{2}}\left[\mu^{2}\left(\frac{\mu+2}{r^{2}}+\frac{6 M}{r^{3}}\right)+\frac{36 M^{2}}{r^{4}}\left(\mu+\frac{2 M}{r}\right)\right]$.

This is an example of Faddeev's theorem [7], which we described in [1], where starting from the original constrained variables $\left(p_{i}, q^{i}\right)$ with constraints $\phi^{\alpha}(p, q)$ one can perform a canonical transformation with $p_{\alpha}^{\prime}=\chi_{\alpha}(p, q)=0$ and $q^{\prime \alpha}=q^{\prime \alpha}\left(p^{*}, q^{*}\right)$ so that the independent set of canonical variables is $\left(p^{*}, q^{*}\right)$. In this particular case we find just one set of $\left(p^{*}, q^{*}\right)$, which are the Zerilli-Moncrief function $\Psi$ of the original variables, and its canonical conjugate.

\section{B. $\ell \geq 2$ odd}

There are three fields in this sector: $h_{a}^{\ell m(-)}, h_{2}^{\ell m(-)}$. In the Regge-Wheeler gauge

$$
h_{2}^{\ell m(-)}=0 .
$$

Equations of motion for the remaining two fields are

$$
\begin{aligned}
P^{t} & =-\frac{\partial^{2}}{\partial t \partial r} h_{r}+\frac{\partial^{2}}{\partial r^{2}} h_{t}-\frac{2}{r} \frac{\partial}{\partial t} h_{r}-\frac{\lambda r-4 M}{r^{3} f} h_{t}, \\
P^{r} & =\frac{\partial^{2}}{\partial t^{2}} h_{r}-\frac{\partial^{2}}{\partial t \partial r} h_{t}+\frac{2}{r} \frac{\partial}{\partial t} h_{t}+\frac{\mu f}{r^{2}} h_{r} .
\end{aligned}
$$

Restoring the quadratic Lagrangian and using partial integration one can identify one field that enters into the Lagrangian without a time derivative: this is $h_{t}$,

$$
\begin{aligned}
\mathcal{L}= & h_{t}\left(-\frac{\partial^{2}}{\partial t \partial r} h_{r}+\frac{1}{2} \frac{\partial^{2}}{\partial r^{2}} h_{t}-\frac{2}{r} \frac{\partial}{\partial t} h_{r}-\frac{1}{2} \frac{\lambda r-4 M}{r^{3} f} h_{t}\right) \\
& +\frac{1}{2} h_{r}\left(\frac{\partial^{2}}{\partial t^{2}} h_{r}+\frac{\mu f}{r^{2}} h_{r}\right) .
\end{aligned}
$$

Thus we find

$$
p_{t}=0,
$$

$$
p_{r}=h_{t, r}-h_{r, t}-\frac{2}{r} h_{t}
$$

and there is a constraint for $h_{t}$ algebraically related to $p_{r}$,

$$
\left(\partial_{r}+\frac{2}{r}\right)\left(p_{r}+h_{t, r}-\frac{2}{r} h_{t}\right)-h_{t, r r}+\frac{\lambda r-4 M}{r^{3} f} h_{t}=0 .
$$

Therefore there is one independent degree of freedom $\left(h_{r}, p_{r}\right)$. These are Faddeev's $\left(p^{*}, q^{*}\right)$ variables, exactly one set in agreement with the counting given above. One can write the corresponding Hamiltonian $H\left(h_{r}, p_{r}\right)$ and the field equations.

On the other hand, the Hamiltonian for this system was already derived in [12] in the framework of the Arnowitt, Deser, Misner construction. The field equations were derived in [13], where the corresponding CunninghamPrice-Moncrief function was introduced. In the notation of [4], this function is

$$
\Psi_{\text {odd }}^{l m}=\frac{2 r}{(\ell-1)(\ell+2)}\left(h_{t, r}^{\ell m}-h_{r, t}^{\ell m}-\frac{2}{r} h_{t}^{\ell m}\right) .
$$


This function in terms of canonical variables above depends on $\left(p_{r}, h_{r}\right)$. As in the even case discussed above we are led to a single field equation for the CunninghamPrice-Moncrief function

$$
\left(\square-V_{\text {odd }}\right) \Psi_{\text {odd }}=S_{\text {odd }} .
$$

Here the expressions for $V_{\text {odd }}$ and $S_{\text {odd }}$ are given in [4], where also the relation between the Cunningham-PriceMoncrief function and the original Regge-Wheeler function is explained. With $\Psi_{\text {odd }} \equiv Q_{\text {odd }}$ the Hamiltonian is

$$
\begin{aligned}
H_{\ell \geq 2, \mathrm{odd}}= & \frac{1}{2} \sum_{\ell \geq 2, m} \int\left[d r f\left(P^{\ell, m}\right)_{\mathrm{odd}}^{2}+f\left(Q_{, r}^{\ell, m}\right)_{\mathrm{odd}}^{2}\right. \\
& \left.+\left(\frac{\ell(\ell+1)}{r^{2}}-\frac{6 M}{r^{3}}\right)\left(Q^{\ell, m}\right)_{\mathrm{odd}}^{2}\right] .
\end{aligned}
$$

\section{QUADRATIC LAGRANGIAN/HAMILTONIAN FOR $\ell<2$ MODES}

\section{A. $\ell=1$ even}

Our six fields are $h_{a b}^{1 m(+)}, j_{a}^{1 m(+)}, K^{1 m(+)}$. We take a gauge-fixing condition [1]

$$
j_{a}^{1 m(+)}=K^{1 m(+)}=0 .
$$

The remaining fields $h_{a b}^{1 m(+)}$ in this gauge satisfy the field equations

$$
\begin{aligned}
Q^{t t} & =\frac{f}{r} \frac{\partial}{\partial r} h_{r r}+\frac{2(r+M)}{r^{3}} h_{r r}, \\
Q^{t r} & =-\frac{f}{r} \frac{\partial}{\partial t} h_{r r}-\frac{1}{r^{2}} h_{t r}, \\
Q^{r r} & =\frac{2 f}{r} \frac{\partial}{\partial t} h_{t r}-\frac{f}{r} \frac{\partial}{\partial r} h_{t t}+\frac{r+2 M}{r^{3}} h_{t t}-\frac{f^{2}}{r^{2}} h_{r r} .
\end{aligned}
$$

We can therefore reconstruct the Lagrangian of the form (1.6) which will produce these equations,

$\mathcal{L}=h_{t t} Q^{t t}+\left(\frac{\partial}{\partial t} h_{t r}\right) \frac{2 f}{r} h_{r r}-h_{t r} \frac{1}{2 r^{2}} h_{t r}-h_{r r} \frac{f^{2}}{2 r^{2}} h_{r r}$.

We now define $q \equiv h_{t r}, p \equiv \frac{2 f}{r} h_{r r}$, and $h_{t t} \equiv \lambda$,

$$
\mathcal{L}=\dot{q} p+\lambda Q^{t t}\left(p, \partial_{r} p\right)-q^{2} \frac{1}{2 r^{2}}-\frac{1}{8} p^{2} .
$$

We integrate out the Lagrange multiplier and find

$$
\mathcal{L}=\dot{q} p-q^{2} \frac{1}{2 r^{2}}-\frac{1}{8} p^{2},
$$

where

$$
\frac{f}{r} \frac{\partial}{\partial r} \frac{r p}{2 f}+\frac{2(r+M)}{r^{3}} \frac{r p}{2 f}=0 \Rightarrow p_{, r}+F(r) p=0 .
$$

The algebraic constraint which $p$ has to satisfy contradicts the commutation relation which has to be imposed for quantization, as shown in Eq. (1.8). There is no solution of the algebraic constraint (4.5) for the canonical momentum $p(t, r)$ that would be consistent with the quantization condition; only $p=0$ is a consistent one. We conclude there that there are no physical degrees of freedom left in this sector,

$$
H_{\ell=1, \text { even }}=0 .
$$

This is in agreement with the counting we presented above.

\section{B. $\ell=1$ odd}

There are two fields: $h_{a}^{1 m(-)}$. We take a gauge-fixing condition $h_{r}^{1 m(-)}=0$ [1]. In this gauge the remaining field equation is

$$
P^{t}=\frac{\partial^{2}}{\partial r^{2}} h_{t}-\frac{2}{r^{2}} h_{t}
$$

The Lagrangian that will generate this equation is

$$
\mathcal{L}=\frac{1}{2} h_{t}\left(\frac{\partial^{2}}{\partial r^{2}} h_{t}-\frac{2}{r^{2}} h_{t}\right) .
$$

There is one field here where the Lagrangian $\mathcal{L}(q)$ does not have the time derivative of this field, and therefore $p=\frac{\delta \mathcal{L}}{\dot{h}_{t}}=0$. There are no canonical variables here and the Hamiltonian vanishes

$$
H_{\ell=1, \text { odd }}=0 .
$$

This is in agreement with the counting we presented above.

\section{C. $\ell=0$ even}

There are four fields here: $h_{a b}^{00(+)}, K^{00(+)}$. We take gaugefixing conditions $K=h_{t r}=0$ [1]. The remaining field equations are

$$
\begin{aligned}
Q^{t t} & =\frac{f}{r} \frac{\partial}{\partial r} h_{r r}+\frac{r+2 M}{r^{3}} h_{r r}, \\
Q^{r r} & =-\frac{f}{r} \frac{\partial}{\partial r} h_{t t}+\frac{2 M}{r^{3}} h_{t t}-\frac{f^{2}}{r^{2}} h_{r r} .
\end{aligned}
$$

The Lagrangian that will generate these equations is 


$$
\mathcal{L}=h_{t t}\left(\frac{f}{r} \frac{\partial}{\partial r} h_{r r}+\frac{(r+2 M)}{r^{3}} h_{r r}\right)-\frac{f^{2}}{2 r^{2}} h_{r r}^{2} .
$$

There are two fields, $q^{1}, q^{2}$, but there are no time derivatives in the Lagrangian, $p_{1}=p_{2}=0$, no canonical variables, and the Hamiltonian vanishes,

$$
H_{\ell=0}=0
$$

This is again in agreement with the counting we presented above.

\section{A SPECIAL ROLE OF $\ell=0,1$ IN QUANTIZATION OF GRAVITY}

Is there any relation between the well-known fact about the absence of radiation from monopoles and dipoles in gravity and the fact we observed here, that there are no quantum physical degrees of freedom in monopoles and dipoles when gravity is quantized in spherical harmonics basis? The answer is yes, and it has to do with the tensor nature of gravity, so that radiation starts with quadrupoles $\ell \geq 2$.

Regge-Wheeler ansatz for $\ell \geq 2$ has ten functions depending on the coordinates of $\mathcal{M}_{2}$ listed in Eqs. (2.2)-(2.6). Here we show them in the matrix form contracted with spherical functions:

$$
\begin{aligned}
h_{\mu \nu}^{\ell>1}= & \left(\begin{array}{cc}
h_{a b}^{\ell m} Y^{\ell m} & j_{a}^{\ell m} Y_{B}^{\ell m} \\
j_{a}^{\ell m} Y_{B}^{\ell m} & r^{2} K^{\ell m} \Omega_{A B} Y^{\ell m}+G^{\ell m} Y_{A B}^{\ell m}
\end{array}\right)^{(+)} \\
& +\left(\begin{array}{cc}
0 & h_{a}^{\ell m} X_{B}^{\ell m} \\
h_{a}^{\ell m} X_{B}^{\ell m} & h_{2}^{\ell m} X_{A B}^{\ell m}
\end{array}\right)^{(-)} .
\end{aligned}
$$

The number gauge symmetries in all cases with $\ell>0$ is the same since $\xi_{\mu}$ is a vector

$$
\xi_{\mu}^{\ell>0}=\left(\begin{array}{c}
\xi_{a}^{\ell m} Y^{\ell m} \\
\xi^{\ell m} Y_{A}^{\ell m}
\end{array}\right)^{(+)}+\left(\begin{array}{c}
0 \\
\xi_{a}^{\ell m} X_{B}^{\ell m}
\end{array}\right)^{(-)} .
$$

Therefore we find that instead of ten fields (even and odd) as for $\ell \geq 2$ we have eight fields (even and odd) for $\ell=1$, no fields in red,

$$
\begin{aligned}
h_{\mu \nu}^{\ell=1}= & \left(\begin{array}{cc}
h_{a b}^{\ell m} Y^{\ell m} & j_{a}^{\ell m} Y_{B}^{\ell m} \\
j_{a}^{\ell m} Y_{B}^{\ell m} & r^{2} K^{\ell m} \Omega_{A B} Y^{\ell m}
\end{array}\right)^{(+)} \\
& +\left(\begin{array}{cc}
0 & h_{a}^{\ell m} X_{B}^{\ell m} \\
h_{a}^{\ell m} X_{B}^{\ell m} & 0
\end{array}\right)^{(-)} .
\end{aligned}
$$

Therefore from $10-2=8$ states we subtract a double set of four symmetries, and find no degrees of freedom for $\ell=1$ since $8-8=0$.
At $\ell=0 Y_{A B}^{00}=X_{A B}^{00}=0$, the terms in red are absent, but also at $Y_{A}^{00}=X_{A}^{00}=0$, all blue terms are absent:

$h_{\mu \nu}^{\ell=0}=\left(\begin{array}{cc}h_{a b}^{\ell m} Y^{\ell m} & 0 \\ 0 & r^{2} K^{\ell m} \Omega_{A B} Y^{\ell m}\end{array}\right)^{(+)}+\left(\begin{array}{ll}0 & 0 \\ 0 & 0\end{array}\right)^{(-)}$,

$$
\xi_{\mu}^{\ell=0}=\left(\begin{array}{c}
\xi_{a}^{\ell m} Y^{\ell m} \\
0
\end{array}\right)^{(+)}+\left(\begin{array}{l}
0 \\
0
\end{array}\right)^{(-)}
$$

We are left with four fields and two gauge symmetries, and there are no degrees of freedom for $\ell=0: 4-4=0$.

\section{QUANTIZATION OF GRAVITY IN SPHERICAL HARMONICS BASIS IN THE FLAT BACKGROUND}

The procedure of Lagrangian quantization performed in [1] as well as the values of the unitary quadratic Hamiltonians presented in this paper have a smooth limit from the Schwarzschild background to a flat one. In Schwarzschild coordinates this means that the limit $M \rightarrow 0$ is regular.

In particular, the Zerilli-Moncrief function for $\ell \geq 2$ in the Regge-Wheeler gauge in the limit $M \rightarrow 0$ is

$\Psi_{\text {even }}^{\ell m}=\frac{2 r}{\ell(\ell+1)}\left[K+\frac{2}{(\ell-1)(\ell+2)}\left(h_{r r}-r K_{, r}\right)\right], \quad \ell \geq 2$.

The Cunningham-Price-Moncrief function is

$\Psi_{\text {odd }}^{\ell m}=\frac{2 r}{(\ell-1)(\ell+2)}\left(h_{t, r}^{\ell m}-h_{r, t}^{\ell m}-\frac{2}{r} h_{t}^{\ell m}\right), \quad \ell \geq 2$.

The quadratic part of the Hamiltonian in both cases is

$$
\begin{aligned}
H_{\text {even/odd }}= & \frac{1}{2} \sum_{\ell \geq 2, m} \int\left[d r\left(P^{\ell, m}\right)_{\text {even } / \text { odd }}^{2}+\left(Q_{, r}^{\ell, m}\right)_{\text {even } / \text { odd }}^{2}\right. \\
& \left.+\frac{\ell(\ell+1)}{r^{2}}\left(Q^{\ell, m}\right)_{\text {even } / \text { odd }}^{2}\right]
\end{aligned}
$$

Here $Q_{\text {even/odd }}=\Psi_{\text {even/odd }}$ and $P_{\text {even/odd }}$ is the corresponding canonical conjugate. At the quadratic level these are the only two physical states that appear in the unitary Hamiltonian.

The higher order terms in each of the quantized actions, at the black hole background, and in the flat background still have to be constructed. 


\section{A COMMENT ON REGGE-WHEELER AND TEUKOLSKY FORMALISM AND GRAVITY WAVES}

The Cunningham-Price-Moncrief (CPM) master function and the Zerilli-Moncrief (ZM) master function, which were identified here as canonical variables in the gravity, appear to play some role also in a more interesting case of the Kerr black holes. Namely, as pointed out in a review [14], there is a relation via Chandrasekhar transformation between these functions and Teukolsky radial function. Note that Teukolsky equations for the Weyl tensor components use the expansion in terms of the spin-weighted spheroidal harmonics. Such an expansion for the metric starts with $\ell=2$.

There is also an interesting relation between the metric perturbation far from the source and our canonical variables in the generalized Regge-Wheeler gauge. Namely, according to [14] the gravitational wave strain can be determined directly from CPM and ZM functions of the metric. Using the Chandrasekhar transformation between these functions and Teukolsky radial function, and some properties of $\psi_{4}=C_{n \bar{m} n \bar{m}}$, the gravitational strain was given as

$r\left(h_{+}-i h_{x}\right)=\sum_{\ell \geq 2} \sum_{|m| \leq \ell} \frac{D}{2}\left(\Psi_{\text {even }}^{\ell m}-i \Psi_{\text {even }}^{\ell m}\right)_{-2} Y_{\ell, m}(\theta, \phi)$,

where ${ }_{-2} Y_{\ell, m}(\theta, \phi)$ is the spin-weighted spheroidal harmonic. That equality holds in the limit $r \rightarrow \infty$ (at fixed $\left.u=t-r_{*}\right)$. Here the constant

$$
D=\sqrt{(\ell-1)(\ell+1)(\ell+1)}
$$

is the Schwarzschild limit of the constant that appears in the Teukolsky-Starobinsky identities. Clearly, the cases $\ell=0,1$ drop from the formula for the gravitational waves. This is in agreement with the fact established in this paper that these modes have no physical degrees of freedom.

\section{SUMMARY}

In this article we have counted the number of physical quantized degrees of freedom of Einstein gravity in the spherical harmonic basis using the standard formula: this number is given by $n-k$, where $n+k$ is the number of components of gauge fields and the gauge theory has $k$ gauge symmetries. For example, in $4 \mathrm{D}$ the graviton has $n+k=6+4=10$ components, and there are $k=4$ gauge symmetries. The number of physical degrees of freedom is $n-k=(n+k)-2 k=10-8=2$.
In the spherical harmonic basis we have found that for each $\ell, m$ in $\ell \geq 2$ sector there is 1 degree of freedom for even parity states and 1 degree of freedom for odd parity states. In the $\ell<2$ sector of gravity we have found that there are no physical degrees of freedom.

To construct the Hamiltonian we start with the ReggeWheeler formulation [2-4] of Einstein gravity in the spherical harmonic basis in the background of a Schwarzschild black hole. The part of the action $S(g+h)$ quadratic in perturbations $h_{\mu \nu}$ in Eq. (1.6) can be presented in the spherical harmonic basis using the explicit form of equations of motion linear in perturbations, as shown in Eq. (1.7). We take these explicit expressions $Q^{\mu \nu}=\frac{\delta S(g, h)}{\delta h_{\mu \nu}}$, which are linear in $h_{\mu \nu}$, from [4], and reconstruct the part of the action $S(g+h)$ quadratic in perturbations $h_{\mu \nu}$. We impose the generalized Regge-Wheeler gauge [1]. The action quadratic in fields we take in Schwarzschild coordinates and proceed with canonical quantization, defining canonical momenta and constraints.

For $\ell \geq 2$ fields the procedure leads to 1 independent degree of freedom for even and 1 for odd modes in each case with $\ell, m$, in agreement with the counting of physical degrees of freedom. We conclude that up to a canonical transformation such a Hamiltonian is equivalent to the one presented in [12] where the corresponding canonical variables are a Zerilli-Moncrief function [3,12] for even modes and a Cunningham-Price-Moncrief function [13] for odd modes. In [12] the modes with $\ell<2$ were not studied.

We apply our method also for $\ell<2$ modes. In each sector for $\ell=1$, even and odd cases, and for $\ell=0$ we first reproduce the action from the explicit expressions $Q^{\mu \nu}=$ $\frac{\delta S(g, h)}{\delta h_{\mu \nu}}$ linear in $h_{\mu \nu}$. We use the gauge-fixing condition for low multipoles in [1] and identify the canonical variables and constraints. In each case the conclusion is that there are no independent unconstrained canonical variables suitable for the quantized Hamiltonian. This is again in agreement with the counting of degrees of freedom performed earlier.

The original goal of this investigation was to develop a consistent method of quantization of a gravitational field in the background of a Schwarzschild black hole [1]. However, we found that in Schwarzschild coordinates the limit $M \rightarrow 0$ is regular, and therefore the quantization procedure is valid in the Minkowski background as well. In this paper we found the Hamiltonian describing unitary evolution of gravitational perturbations in spherical coordinates, which equally well applies to quantization of gravity in Minkowski background as well as in the Schwarzschild black hole background. The choice of the generalized Regge-Wheeler gauge in [1] where the gravity 
Hamiltonian is unitary requires one to use the spherical harmonic basis for the metric perturbations. This unitary gauge is a Regge-Wheeler gauge $G^{\ell m(+)}=j_{a}^{\ell m(+)}=$ $h_{2}^{\ell m(-)}=0$ for $\ell \geq 2$. For $\ell=1$ it is $j_{a}^{1 m(+)}=$ $K^{1 m(+)}=h_{r}^{1 m(-)}=0$, and for $\ell=0$ it is $K^{00}=h_{t r}^{00}=0$.

In this generalized Regge-Wheeler gauge, the quadratic part of the Hamiltonian for $\ell<2$ modes is vanishing, whereas for $\ell \geq 2$ it is given in Eqs. (3.6) and (3.15) in the black hole background and in Eq. (6.3) in the Minkowski background.

\section{ACKNOWLEDGMENTS}

I am grateful to A. Barvinsky, E. Coleman, A. Linde, E. Poisson, A. Rahman, P. Stamp, A. Starobinsky, A. Vainshtein, A. Van Proeyen, and I. Volovich for stimulating and helpful discussions. I am supported by the Stanford Institute of Theoretical Physics and by the Grant No. PHY2014215 of National Science Foundation (United States/ US), and by the Simons Foundation Origins of the Universe program (Modern Inflationary Cosmology collaboration (United States/US)).
[1] R. Kallosh and A. A. Rahman, Quantization of gravity in the black hole background, Phys. Rev. D 104, 086008 (2021).

[2] T. Regge and J. A. Wheeler, Stability of a Schwarzschild singularity, Phys. Rev. 108, 1063 (1957).

[3] F. Zerilli, Gravitational field of a particle falling in a schwarzschild geometry analyzed in tensor harmonics, Phys. Rev. D 2, 2141 (1970).

[4] K. Martel and E. Poisson, Gravitational perturbations of the Schwarzschild spacetime: A practical covariant and gauge-invariant formalism, Phys. Rev. D 71, 104003 (2005).

[5] B. S. DeWitt, Quantum theory of gravity. 2. The manifestly covariant theory, Phys. Rev. 162, 1195 (1967).

[6] L. Faddeev and V. Popov, Feynman diagrams for the Yang-Mills field, Phys. Lett. 25B, 29 (1967).

[7] L. D. Faddeev, Feynman integral for singular Lagrangians, Theor. Math. Phys. 1, 1 (1969).

[8] E. S. Fradkin and I. V. Tyutin, S matrix for Yang-Mills and gravitational fields, Phys. Rev. D 2, 2841 (1970).
[9] L. D. Faddeev and V. N. Popov, Covariant quantization of the gravitational field, Sov. Phys. Usp. 16, 777 (1974).

[10] E. S. Fradkin and G. A. Vilkovisky, Quantization of relativistic systems with constraints: Equivalence of canonical and covariant formalisms in quantum theory of gravitational field, Lebedev Physical Institute Report No. CERN-TH2332, 1977.

[11] I. A. Batalin and G. A. Vilkovisky, Relativistic S matrix of dynamical systems with boson and fermion constraints, Phys. Lett. 69B, 309 (1977).

[12] V. Moncrief, Gravitational perturbations of spherically symmetric systems. I. The exterior problem, Ann. Phys. (N.Y.) 88, 323 (1974).

[13] C. T. Cunningham, R. H. Price, and V. Moncrief, Radiation from collapsing relativistic stars. I-Linearized odd-parity radiation, Astrophys. J. 224, 643 (1978).

[14] A. Pound and B. Wardell, Black hole perturbation theory and gravitational self-force, arXiv:2101.04592. 